\title{
Removable sets for subcaloric functions and solutions of semilinear heat equations with absorption
}

\author{
Kentaro HiRATA
}

(Received January 17, 2014; Revised September 8, 2014)

\begin{abstract}
We investigate removable sets for subcaloric functions satisfying either a growth condition or an integrability condition by defining suitably upper Minkowski content with respect to the parabolic distance. Results are also applied to obtain removability theorems for nonnegative solutions of a semilinear heat equation with an absorption term.
\end{abstract}

Key words: removable singularity, subcaloric function, caloric function, semilinear heat equation, Minkowski content, Hausdorff measure.

\section{Introduction}

It is well known that any closed set in $\mathbb{R}^{n}$ of capacity zero is removable for subharmonic functions which are bounded above. Moreover, the Hausdorff dimension of such a set is at most $n-2$. When the size of an exceptional set is small in some sense, the boundedness condition for functions can be weaken. In [3], Gardiner gave removability theorems by noting a relation between the Hausdorff measure of an exceptional set $E$ and a growth rate of subharmonic functions near $E$ when $E$ lies in an $(n-1)$-dimensional smooth manifold in $\mathbb{R}^{n}$. Later, Riihentaus [9] removed smoothness conditions on $E$ by considering upper Minkowski content and developing arguments in Harvey and Polking [4].

For the heat equation, it is also known that any closed set in $\mathbb{R}^{n+1}$ of thermal capacity zero is removable for subcaloric functions which are bounded above and that the Hausdorff dimension of it is at most $n$. See Watson [13] and Taylor and Watson [11]. Here we should note that the definition of Hausdorff dimension in this case is different from the usual one and is given in terms of the parabolic distance. Recently, Hsu [7] and Hui

2000 Mathematics Subject Classification : Primary 35B65; Secondary 31C05, 35K05, $35 \mathrm{~K} 91$.

The author was partially supported by Grant-in-Aid for Scientific Research (C) (No. 26400137), Japan Society for the Promotion of Science. 
[8] proved that the set $\{0\} \times(0, T)$ in $\mathbb{R}^{n} \times \mathbb{R}$, where $n \geq 3$, is removable for caloric functions satisfying the growth condition: for any closed interval $I \subset(0, T)$,

$$
\max _{t \in I}|u(x, t)|=o\left(\|x\|^{2-n}\right) \quad \text { as } x \rightarrow 0 .
$$

Motivated by the removability of moving singularities, Takahashi and Yanagida [10] extended this result to the case that exceptional sets lie in curves or, more generally, low-dimensional surfaces moving smoothly in the spatial directions and 1/2-Hölder continuously in the temporal direction with respect to the usual Euclidean distance.

The purpose of this note is to present parabolic analogs of Riihentaus' results by introducing upper Minkowski content with respect to the parabolic distance, that is, to examine removable sets for subcaloric functions satisfying either a growth condition or an integrability condition. Our result extends Takahashi and Yanagida's in some directions: to subcaloric functions and to more general removable sets. As applications, we also obtain removability theorems for positive solutions of a semilinear heat equation with an absorption term.

\section{Notations and results}

To state our results, we introduce upper Minkowski content with respect to the parabolic distance. Let us denote a typical point in $\mathbb{R}^{n+1}$ by a bold letter like $\boldsymbol{x}=(x, t)$, where $x \in \mathbb{R}^{n}$, a spatial variable, and $t \in \mathbb{R}$, a temporal variable. The parabolic distance between two points $\boldsymbol{x}=(x, t)$ and $\boldsymbol{y}=$ $(y, s)$ is defined by

$$
d_{\mathrm{p}}(\boldsymbol{x}, \boldsymbol{y}):=\|x-y\|+|t-s|^{1 / 2},
$$

where $\|\cdot\|$ is the Euclidean norm on $\mathbb{R}^{n}$. In all notations, the subscript "p" means that they are relative to a "parabolic" content. Let $E$ be a set in $\mathbb{R}^{n+1}$ and let $r>0$. Denoting the parabolic distance from a point $\boldsymbol{x}$ to $E$ by $d_{\mathrm{p}}(\boldsymbol{x}, E)$, we define an $r$-neighborhood of $E$ by

$$
E(r):=\left\{\boldsymbol{x} \in \mathbb{R}^{n+1}: d_{\mathrm{p}}(\boldsymbol{x}, E)<r\right\} .
$$

Let $0 \leq \alpha \leq n+2$. We define the $\alpha$-dimensional upper Minkowski content 
of $E$ with respect to the parabolic distance $d_{\mathrm{p}}$ by

$$
\mathcal{M}_{\mathrm{p}}^{\alpha}(E):=\limsup _{r \rightarrow 0} \frac{\mathfrak{m}(E(r))}{r^{n+2-\alpha}}
$$

where $\mathfrak{m}$ denotes Lebesgue measure on $\mathbb{R}^{n+1}$. Note that the power of the denominator of the right hand side is not $n+1-\alpha$, because the temporal direction is actually two dimensional. Let $\Omega$ be an open set in $\mathbb{R}^{n+1}$. A function $u: \Omega \rightarrow[-\infty,+\infty)$ is called subcaloric on $\Omega$ if $u$ is upper semicontinuous on $\Omega, u$ is finite on a dense subset of $\Omega$, and $u$ satisfies the submean value inequality: for any $\boldsymbol{x}=(x, t) \in \Omega$ and small $0<r<r_{\boldsymbol{x}}$,

$$
u(\boldsymbol{x}) \leq \frac{1}{2^{n+2}(\pi r)^{n / 2}} \int_{B_{\mathrm{p}}(\boldsymbol{x}, r)} \frac{\|x-y\|^{2}}{(t-s)^{2}} u(y, s) \mathrm{d} \mathfrak{m}(y, s),
$$

where the heat ball of center $\boldsymbol{x}$ and radius $r$ is defined by

$$
B_{\mathrm{p}}(\boldsymbol{x}, r):=\left\{(y, s): s<t, \frac{1}{(t-s)^{n / 2}} \exp \left(-\frac{\|x-y\|^{2}}{4(t-s)}\right)>\frac{1}{r^{n / 2}}\right\} .
$$

Throughout this note (except in Appendix), we suppose that $\Omega$ is an open subset of $\mathbb{R}^{n+1}$.

Theorem 2.1 Let $0 \leq \alpha<n$, let $E$ be a relatively closed subset of $\Omega$, and let $u$ be a subcaloric function on $\Omega \backslash E$. Assume one of the following:

(i) $\mathcal{M}_{\mathrm{p}}^{\alpha}(E)=0$ and

$$
\limsup _{\Omega \backslash E \ni \boldsymbol{x} \rightarrow \boldsymbol{y}} d_{\mathrm{p}}(\boldsymbol{x}, E)^{n-\alpha} u(\boldsymbol{x})<\infty \quad \text { for each } \boldsymbol{y} \in E
$$

(ii) $\mathcal{M}_{\mathrm{p}}^{\alpha}(E)<\infty$ and

$$
\limsup _{\Omega \backslash E \ni \boldsymbol{x} \rightarrow \boldsymbol{y}} d_{\mathrm{p}}(\boldsymbol{x}, E)^{n-\alpha} u(\boldsymbol{x}) \leq 0 \quad \text { for each } \boldsymbol{y} \in E
$$

Then $u$ has a subcaloric extension to $\Omega$.

By a $C^{2,1}$-function, we mean a function which is twice differentiable with respect to the spatial variables and differentiable with respect to the temporal variable and whose all partial derivatives are continuous. We say 
that a real-valued function $u$ is caloric on $\Omega$ if $u$ is a $C^{2,1}$-function on $\Omega$ satisfying the heat equation $\Delta u-\partial u / \partial t=0$ in $\Omega$, where $\Delta$ is the Laplacian on $\mathbb{R}^{n}$. This is equivalent to that both of $u$ and $-u$ are subcaloric on $\Omega$. As a consequence of Theorem 2.1, we obtain the following.

Corollary 2.2 Let $0 \leq \alpha<n$, let $E$ be a relatively closed subset of $\Omega$, and let $u$ be a caloric function on $\Omega \backslash E$. Assume one of the following:

(i) $\mathcal{M}_{\mathrm{p}}^{\alpha}(E)=0$ and

$$
\limsup _{\Omega \backslash E \ni \boldsymbol{x} \rightarrow \boldsymbol{y}} d_{\mathrm{p}}(\boldsymbol{x}, E)^{n-\alpha}|u(\boldsymbol{x})|<\infty \quad \text { for each } \boldsymbol{y} \in E ;
$$

(ii) $\mathcal{M}_{\mathrm{p}}^{\alpha}(E)<\infty$ and

$$
\lim _{\Omega \backslash E \ni \boldsymbol{x} \rightarrow \boldsymbol{y}} d_{\mathrm{p}}(\boldsymbol{x}, E)^{n-\alpha}|u(\boldsymbol{x})|=0 \quad \text { for each } \boldsymbol{y} \in E .
$$

Then $u$ has a caloric extension to $\Omega$.

Remark 2.3 In Appendix, we will show that an exceptional set $E$ considered in [10] satisfies $\mathcal{M}_{\mathrm{p}}^{\alpha}(E)<\infty$ for some positive integer $\alpha<n$. Thus Corollary 2.2 (ii) extends a result in [10].

The Minkowski content condition on removable sets can be weaken when we target higher order integrable subcaloric functions instead of those satisfying the above growth condition. To this end, we recall Hausdorff (outer) measure on $\left(\mathbb{R}^{n+1}, d_{\mathrm{p}}\right)$. By a parabolic cube of center $\boldsymbol{x}=\left(x_{1}, \ldots, x_{n}, t\right)$ and side length $r$, we mean a closed cube in $\mathbb{R}^{n+1}$ of the form

$$
\prod_{i=1}^{n}\left[x_{i}-\frac{1}{2} r, x_{i}+\frac{1}{2} r\right] \times\left[t-\frac{1}{2} r^{2}, t+\frac{1}{2} r^{2}\right]
$$

and denote it by $Q(\boldsymbol{x}, r)$. For $E \subset \mathbb{R}^{n+1}, \alpha \geq 0$ and $\delta>0$, we let

$$
\mathcal{H}_{\mathrm{p}, \delta}^{\alpha}(E)=\inf \left\{\sum_{i} r_{i}^{\alpha}\right\}
$$

where the infimum is taken over all possible coverings of $E$ by a countable collection of parabolic cubes $\left\{Q\left(\boldsymbol{x}_{i}, r_{i}\right)\right\}$ such that $r_{i} \leq \delta$ for each $i$. Since $\mathcal{H}_{\mathrm{p}, \delta}^{\alpha}(E)$ is a nonincreasing function for $\delta$, the limit 


$$
\mathcal{H}_{\mathrm{p}}^{\alpha}(E):=\lim _{\delta \rightarrow 0} \mathcal{H}_{\mathrm{p}, \delta}^{\alpha}(E)
$$

exists. This is called $\alpha$-dimensional parabolic Hausdorff (outer) measure. It is easy to show that if $E$ is a compact subset of $\mathbb{R}^{n+1}$ with $\mathcal{M}_{\mathrm{p}}^{\alpha}(E)<\infty$, then $\mathcal{H}_{\mathrm{p}}^{\alpha}(E)<\infty$. See Lemma 3.2 below. But the opposite does not hold in general. Moreover, we have $\mathfrak{m}(E)=0$ when $\mathcal{H}_{\mathrm{p}}^{\alpha}(E)<\infty$ or $\mathcal{M}_{\mathrm{p}}^{\alpha}(E)<\infty$ for some $0 \leq \alpha<n+1$. See Lemma 3.1 below.

We introduce an important index. For $0 \leq \alpha<n$, we let

$$
q_{\alpha}:=\frac{n-\alpha+2}{n-\alpha} .
$$

Write $u^{+}:=\max \{u, 0\}$ for short. Also, we denote by $\mathcal{L}_{\text {loc }}^{q}(\Omega)$ the set of all Lebesgue measurable functions $u$ defined on $\Omega$ except for some measurable set $A$ with $\mathfrak{m}(A)=0$ such that $\int_{K \backslash A}|u(\boldsymbol{x})|^{q} \mathrm{~d} \mathfrak{m}(\boldsymbol{x})<\infty$ for any compact subset $K$ of $\Omega$. The case $q=\infty$ is defined in a similar way.

Theorem 2.4 Let $0 \leq \alpha<n$, let $E$ be a relatively closed subset of $\Omega$ such that $\mathcal{H}_{\mathrm{p}}^{\alpha}(E)<\infty$, and let $u$ be a subcaloric function on $\Omega \backslash E$. If $u^{+} \in$ $\mathcal{L}_{\text {loc }}^{q_{\alpha}}(\Omega)$, then $u$ has a subcaloric extension to $\Omega$.

The next corollary follows immediately from Theorem 2.4.

Corollary 2.5 Let $0 \leq \alpha<n$, let $E$ be a relatively closed subset of $\Omega$ such that $\mathcal{H}_{\mathrm{p}}^{\alpha}(E)<\infty$, and let $u$ be a caloric function on $\Omega \backslash E$. If $u \in \mathcal{L}_{\text {loc }}^{q_{\alpha}}(\Omega)$, then $u$ has a caloric extension to $\Omega$.

Recently, the author extended a removability theorem for caloric functions proved by Hsu [7] and Hui [8] to solutions of semilinear heat equations with nonlinear terms (see [6]). But there only the exceptional set $\{0\} \times(0, T)$ was considered. Our above results are also applicable to removability theorems for the following heat equation with an absorption term:

$$
\Delta u-\frac{\partial u}{\partial t}=u^{q}
$$

where $q \geq 1$.

Corollary 2.6 Let $0 \leq \alpha<n$, let $E$ be a relatively closed subset of $\Omega$, and let $u$ be a nonnegative $C^{2,1}$-solution of (2.3) in $\Omega \backslash E$. Assume one of the following: 
(i) $\mathcal{M}_{\mathrm{p}}^{\alpha}(E)=0$ and

$$
\limsup _{\Omega \backslash E \ni \boldsymbol{x} \rightarrow \boldsymbol{y}} d_{\mathrm{p}}(\boldsymbol{x}, E)^{n-\alpha} u(\boldsymbol{x})<\infty \quad \text { for each } \boldsymbol{y} \in E
$$

(ii) $\mathcal{M}_{\mathrm{p}}^{\alpha}(E)<\infty$ and

$$
\lim _{\Omega \backslash E \ni \boldsymbol{x} \rightarrow \boldsymbol{y}} d_{\mathrm{p}}(\boldsymbol{x}, E)^{n-\alpha} u(\boldsymbol{x})=0 \quad \text { for each } \boldsymbol{y} \in E .
$$

Then $u$ can be extended to the whole of $\Omega$ as a $C^{2,1}$-solution of (2.3) in $\Omega$.

Corollary 2.7 Let $0 \leq \alpha<n$, let $E$ be a relatively closed subset of $\Omega$ such that $\mathcal{H}_{\mathrm{p}}^{\alpha}(E)<\infty$, and let $u$ be a nonnegative $C^{2,1}$-solution of $(2.3)$ in $\Omega \backslash E$. If $u \in \mathcal{L}_{\text {loc }}^{q_{\alpha}}(\Omega)$, then $u$ can be extended to the whole of $\Omega$ as a $C^{2,1}$-solution of (2.3) in $\Omega$.

If $q \geq q_{\alpha}$, then no additional assumptions on $u$ are needed. The following is a parabolic analog of Véron's result for semilinear elliptic equations [12].

Corollary 2.8 Let $E$ be a relatively closed subset of $\Omega$, let $0 \leq \alpha<n$, let

$$
q \geq q_{\alpha}
$$

and let $u$ be a nonnegative $C^{2,1}$-solution of $(2.3)$ in $\Omega \backslash E$. If $\mathcal{M}_{\mathrm{p}}^{\alpha}(E)<\infty$, then $u$ can be extended to the whole of $\Omega$ as a $C^{2,1}$-solution of (2.3) in $\Omega$.

The plan of this note is as follows. In Section 3, we collect elementary lemmas which will be used in the proofs of the theorems. Theorems 2.1 and 2.4 are proved in Sections 4 and 5, respectively. The proofs of Corollaries 2.6-2.8 are given in Section 6.

\section{Preliminaries}

This section collects elementary lemmas which are used in the subsequent sections. The symbol $C$ stands for an absolute positive constant whose value is unimportant and may vary at each occurrence.

\subsection{Some properties concerning $\mathcal{M}_{\mathrm{p}}^{\alpha}$ and $\mathcal{H}_{\mathrm{p}}^{\alpha}$}

As proved by Taylor and Watson [11] and Watson [13], it is known that a Borel set in $\mathbb{R}^{n+1}$ with zero $n$-dimensional parabolic Hausdorff measure is a polar set and that every polar set has $(n+1)$-dimensional Lebesgue 
measure zero.

Lemma 3.1 Let $E$ be a Borel set in $\mathbb{R}^{n+1}$ satisfying either $\mathcal{M}_{\mathrm{p}}^{\alpha}(E)<\infty$ or $\mathcal{H}_{\mathrm{p}}^{\alpha}(E)<\infty$ for some $0 \leq \alpha<n+1$. Then $\mathfrak{m}(E)=0$.

Proof. By definition, it is trivial that $\mathcal{M}_{\mathrm{p}}^{\alpha}(E)<\infty$ implies $\mathfrak{m}(E)=0$. We consider the case $\mathcal{H}_{\mathrm{p}}^{\alpha}(E)<\infty$. For a parabolic cube $Q(\boldsymbol{x}, r)$ defined by (2.2), we write

$$
\widetilde{Q}(\boldsymbol{x}, r):=\prod_{i=1}^{n}\left[x_{i}-\frac{1}{2} r, x_{i}+\frac{1}{2} r\right] \times\left[t-\frac{1}{2} r, t+\frac{1}{2} r\right] .
$$

This is the usual cube of center $\boldsymbol{x}=\left(x_{1}, \ldots, x_{n}, t\right)$ and side length $r$. Let $0<\delta<1$ and let $\left\{Q\left(\boldsymbol{x}_{i}, r_{i}\right)\right\}$ be a covering of $E$ such that $r_{i} \leq \delta$ for each $i$. Then $\left\{\widetilde{Q}\left(\boldsymbol{x}_{i}, r_{i}\right)\right\}$ is also a covering of $E$. It follows that

$$
\mathfrak{m}(E) \leq \sum_{i} r_{i}^{n+1} \leq \delta^{n+1-\alpha} \sum_{i} r_{i}^{\alpha}
$$

and so $\mathfrak{m}(E) \leq \delta^{n+1-\alpha} \mathcal{H}_{\mathrm{p}}^{\alpha}(E)$. Letting $\delta \rightarrow 0$, we obtain $\mathfrak{m}(E)=0$.

Lemma 3.2 Let $0 \leq \alpha \leq n+2$. Then there exists a positive constant $C$ depending only on $\alpha$ and $n$ such that for any compact subset $E$ of $\mathbb{R}^{n+1}$,

$$
\mathcal{H}_{\mathrm{p}}^{\alpha}(E) \leq C \mathcal{M}_{\mathrm{p}}^{\alpha}(E)
$$

Proof. We may assume that $\mathcal{M}_{\mathrm{p}}^{\alpha}(E)<\infty$. Let $\kappa>\mathcal{M}_{\mathrm{p}}^{\alpha}(E)$. By definition, we have $\mathfrak{m}(E(r)) \leq \kappa r^{n+2-\alpha}$ for small $r>0$. Let $\delta>0$ be sufficiently small. By the compactness of $E$ and the basic covering lemma, we find finitely many points $\left\{\boldsymbol{x}_{i}\right\}_{i=1}^{N}$ in $E$ such that $\left\{Q\left(\boldsymbol{x}_{i}, \delta\right)\right\}_{i=1}^{N}$ are mutually disjoint and $E \subset \bigcup_{i=1}^{N} Q\left(\boldsymbol{x}_{i}, C_{1} \delta\right)$ for some constant $C_{1}>1$ depending only on $n$. Since $Q\left(\boldsymbol{x}_{i}, \delta\right) \subset E((\sqrt{n}+1) \delta)$, it follows that

$$
\begin{aligned}
N \delta^{n+2} & =\sum_{i=1}^{N} \mathfrak{m}\left(Q\left(\boldsymbol{x}_{i}, \delta\right)\right)=\mathfrak{m}\left(\bigcup_{i=1}^{N} Q\left(\boldsymbol{x}_{i}, \delta\right)\right) \\
& \leq \mathfrak{m}(E((\sqrt{n}+1) \delta)) \leq \kappa((\sqrt{n}+1) \delta)^{n+2-\alpha}
\end{aligned}
$$

and so $N \leq C_{\kappa} \delta^{-\alpha}$. Therefore 


$$
\mathcal{H}_{\mathrm{p}, C_{1} \delta}^{\alpha}(E) \leq \sum_{i=1}^{N}\left(C_{1} \delta\right)^{\alpha} \leq C_{\kappa}
$$

where a constant $C$ depends only on $\alpha$ and $n$. Thus $\mathcal{H}_{\mathrm{p}}^{\alpha}(E) \leq C \kappa$. Letting $\kappa \rightarrow \mathcal{M}_{\mathrm{p}}^{\alpha}(E)$, we obtain the required inequality.

Lemma 3.3 Let $0 \leq \alpha<n$, let $\alpha-n-2<\lambda<0$, and let $E$ be a closed subset of $\mathbb{R}^{n+1}$. Then the following hold:

(i) If $\mathcal{M}_{\mathrm{p}}^{\alpha}(E)=0$, then

$$
\lim _{r \rightarrow 0} r^{\alpha-n-2-\lambda} \int_{E(r)} d_{\mathrm{p}}(\boldsymbol{x}, E)^{\lambda} \mathrm{d} \mathfrak{m}(\boldsymbol{x})=0 .
$$

(ii) If $\mathcal{M}_{\mathrm{p}}^{\alpha}(E)<\infty$, then

$$
\limsup _{r \rightarrow 0} r^{\alpha-n-2-\lambda} \int_{E(r)} d_{\mathrm{p}}(\boldsymbol{x}, E)^{\lambda} \mathrm{d} \mathfrak{m}(\boldsymbol{x})<\infty .
$$

Proof. We show (i) only. Let $\varepsilon>0$. By $\mathcal{M}_{\mathrm{p}}^{\alpha}(E)=0$, there exists $r_{\varepsilon}>0$ such that

$$
\mathfrak{m}(E(r)) \leq \varepsilon r^{n+2-\alpha} \text { for all } 0<r<r_{\varepsilon}
$$

Therefore, for such an $r$,

$$
\begin{aligned}
\int_{E(r)} d_{\mathrm{p}}(\boldsymbol{x}, E)^{\lambda} \mathrm{d} \mathfrak{m}(\boldsymbol{x}) & =r^{\lambda} \mathfrak{m}(E(r))-\lambda \int_{0}^{r} \rho^{\lambda-1} \mathfrak{m}(E(\rho)) \mathrm{d} \rho \\
& \leq \varepsilon r^{\lambda+n+2-\alpha}-\lambda \varepsilon \int_{0}^{r} \rho^{\lambda+n+1-\alpha} \mathrm{d} \rho \\
& \leq C \varepsilon r^{\lambda+n+2-\alpha},
\end{aligned}
$$

which yields (3.2).

Lemma 3.4 The assumptions are the same as in Theorem 2.1. Let $K$ be a compact subset of $E$. Then

$$
\limsup _{r \rightarrow 0} r^{-2} \int_{K(r) \backslash E} u \mathrm{~d} \mathfrak{m} \leq 0 .
$$


Proof. Define

$$
v(\boldsymbol{x}):= \begin{cases}d_{\mathrm{p}}(\boldsymbol{x}, E)^{n-\alpha} u(\boldsymbol{x}) & (\boldsymbol{x} \in \Omega \backslash E), \\ \limsup _{\Omega \backslash E \ni \boldsymbol{y} \rightarrow \boldsymbol{x}} d_{\mathrm{p}}(\boldsymbol{y}, E)^{n-\alpha} u(\boldsymbol{y}) & (\boldsymbol{x} \in E) .\end{cases}
$$

Then $v$ is upper semicontinuous on $\Omega$. Let $K$ be a compact subset of $E$.

First, we consider the case where (i) in Theorem 2.1 holds. For each $i \in \mathbb{N}$, we let

$$
O_{i}:=\{\boldsymbol{x} \in \Omega: v(\boldsymbol{x})<i\}
$$

Then $\left\{O_{i}\right\}$ is an open covering of $K$. Therefore we find $N \in \mathbb{N}$ such that $K \subset \bigcup_{i=1}^{N} O_{i}$, which implies that there exists $r_{1}>0$ such that

$$
u(\boldsymbol{x}) \leq N d_{\mathrm{p}}(\boldsymbol{x}, E)^{\alpha-n} \quad \text { for all } \boldsymbol{x} \in K\left(r_{1}\right) \backslash E .
$$

Let $\varepsilon>0$. It then follows from Lemma 3.3 (i) that for sufficiently small $r>0$,

$$
\int_{K(r) \backslash E} u(\boldsymbol{x}) \mathrm{d} \mathfrak{m}(\boldsymbol{x}) \leq N \int_{E(r)} d_{\mathrm{p}}(\boldsymbol{x}, E)^{\alpha-n} \mathrm{~d} \mathfrak{m}(\boldsymbol{x}) \leq N \varepsilon r^{2} .
$$

Thus we obtain (3.3) in this case.

Next, we consider the case where (ii) in Theorem 2.1 holds. For $\varepsilon>0$, we let $O_{\varepsilon}:=\{\boldsymbol{x} \in \Omega: v(\boldsymbol{x})<\varepsilon\}$. Then $O_{\varepsilon}$ is an open set such that $K \subset O_{\varepsilon}$. Therefore there exists $r_{\varepsilon}>0$ such that

$$
u(\boldsymbol{x}) \leq \varepsilon d_{\mathrm{p}}(\boldsymbol{x}, E)^{\alpha-n} \quad \text { for all } \boldsymbol{x} \in K\left(r_{\varepsilon}\right) \backslash E .
$$

By Lemma 3.3 (ii), we have for $0<r<r_{\varepsilon}$,

$$
\int_{K(r) \backslash E} u(\boldsymbol{x}) \mathrm{d} \mathfrak{m}(\boldsymbol{x}) \leq \varepsilon \int_{E(r)} d_{\mathrm{p}}(\boldsymbol{x}, E)^{\alpha-n} \mathrm{~d} \mathfrak{m}(\boldsymbol{x}) \leq C \varepsilon r^{2},
$$

which concludes (3.3). Thus the lemma is proved.

\subsection{Parabolic dyadic cubes and a partition of unity}

We need to consider dyadic cubes with respect to the parabolic distance. Let $k \in \mathbb{Z}$, the set of integers. In view of the definition of the parabolic 
distance, those are naturally given by

$$
\prod_{i=1}^{n}\left[2^{-k} p_{i}, 2^{-k}\left(p_{i}+1\right)\right] \times\left[2^{-2 k} p_{n+1}, 2^{-2 k}\left(p_{n+1}+1\right)\right]
$$

where $p_{i} \in \mathbb{Z}$ for $i=1, \ldots, n+1$. These sets are called parabolic dyadic cubes of side length $2^{-k}$. Two cubes are said to be disjoint if their intersection has no interior. For a parabolic cube $Q=Q(\boldsymbol{x}, r)$ of center $\boldsymbol{x}=\left(x_{1}, \ldots, x_{n}, t\right)$ and side length $r$, we denote

$$
\frac{3}{2} Q:=\prod_{i=1}^{n}\left[x_{i}-\frac{3}{4} r, x_{i}+\frac{3}{4} r\right] \times\left[t-\frac{3}{4} r^{2}, t+\frac{3}{4} r^{2}\right] .
$$

Note that this is not the parabolic cube in the sense of $(2.2)$ because the temporal direction has length $(3 / 2) r^{2}$, not $((3 / 2) r)^{2}$. As a parabolic analog of [4, Lemma 3.1], we can obtain the following partition of unity subordinate to parabolic dyadic cubes. Write spt $\phi$ for the support of a function $\phi$.

Lemma 3.5 Let $\left\{Q_{i}\right\}_{i=1}^{N}$ be a finite disjoint collection of parabolic dyadic cubes and let $\ell_{i}$ denote the side length of $Q_{i}$. Then there exist nonnegative functions $\left\{\phi_{i}\right\}_{i=1}^{N}$ with the following properties:

- $\phi_{i} \in C_{0}^{\infty}\left(\mathbb{R}^{n+1}\right)$ and $\operatorname{spt} \phi_{i} \subset(3 / 2) Q_{i}$ for $i=1, \ldots, N$;

- $\sum_{i=1}^{N} \phi_{i} \leq 1$ on $\mathbb{R}^{n+1}$ and $\sum_{i=1}^{N} \phi_{i}=1$ on $\bigcup_{i=1}^{N} Q_{i}$;

- there exists a positive constant $C$ depending only on $n$ such that for each $i=1, \ldots, N$,

$$
\left|\frac{\partial \phi_{i}}{\partial x_{1}}\right|, \ldots,\left|\frac{\partial \phi_{i}}{\partial x_{n}}\right| \leq C \ell_{i}^{-1} \quad \text { on } \mathbb{R}^{n+1}
$$

and

$$
\left|\frac{\partial^{2} \phi_{i}}{\partial x_{1}^{2}}\right|, \ldots,\left|\frac{\partial^{2} \phi_{i}}{\partial x_{n}^{2}}\right|,\left|\frac{\partial \phi_{i}}{\partial t}\right| \leq C \ell_{i}^{-2} \quad \text { on } \mathbb{R}^{n+1} \text {. }
$$

Moreover, for each $k=1, \ldots, n$ and $m=1, \ldots, N$,

$$
\left|\frac{\partial}{\partial x_{k}} \sum_{i=1}^{m} \phi_{i}\right| \leq C \max \left\{\ell_{1}^{-1}, \ldots, \ell_{m}^{-1}\right\} \quad \text { on } \mathbb{R}^{n+1}
$$


and

$$
\left|\frac{\partial^{2}}{\partial x_{k}^{2}} \sum_{i=1}^{m} \phi_{i}\right|,\left|\frac{\partial}{\partial t} \sum_{i=1}^{m} \phi_{i}\right| \leq C \max \left\{\ell_{1}^{-2}, \ldots, \ell_{m}^{-2}\right\} \quad \text { on } \mathbb{R}^{n+1} \text {. }
$$

Proof. By relabeling, we may assume that $\ell_{1} \geq \ell_{2} \geq \cdots \geq \ell_{N}$. Let $Q:=[-1 / 2,1 / 2] \times \cdots \times[-1 / 2,1 / 2]$, the cube in $\mathbb{R}^{n+1}$ centered at the origin of side length 1 . Take $\psi \in C_{0}^{\infty}\left(\mathbb{R}^{n+1}\right)$ satisfying $0 \leq \psi \leq 1$ on $\mathbb{R}^{n+1}$, $\psi=1$ on $Q$, and $\psi=0$ outside $(3 / 2) Q$. Denoting the center of $Q_{i}$ by $\left(x_{1}^{(i)}, \ldots, x_{n}^{(i)}, t^{(i)}\right)$, we let

$$
\psi_{i}(\boldsymbol{x}):=\psi\left(\frac{x_{1}-x_{1}^{(i)}}{\ell_{i}}, \ldots, \frac{x_{n}-x_{n}^{(i)}}{\ell_{i}}, \frac{t-t^{(i)}}{\ell_{i}^{2}}\right)
$$

for $\boldsymbol{x}=\left(x_{1}, \ldots, x_{n}, t\right) \in \mathbb{R}^{n+1}$ and $i=1, \ldots, N$. Define $\phi_{1}:=\psi_{1}$ and

$$
\phi_{j}:=\psi_{j} \prod_{i=1}^{j-1}\left(1-\psi_{i}\right) \text { for } j=2, \ldots, N
$$

Then $\phi_{j} \in C^{\infty}\left(\mathbb{R}^{n+1}\right)$ is nonnegative and $\operatorname{spt} \phi_{j} \subset \operatorname{spt} \psi_{j} \subset(3 / 2) Q_{j}$. Also, by an induction argument, we see that for $m=1, \ldots, N$,

$$
\sum_{j=1}^{m} \phi_{j}=1-\prod_{i=1}^{m}\left(1-\psi_{i}\right)
$$

In particular, $\sum_{j=1}^{N} \phi_{j} \leq 1$ on $\mathbb{R}^{n+1}$. Since $\psi_{i}=1$ on $Q_{i}$ for $i=1, \ldots, N$, we have

$$
\sum_{j=1}^{N} \phi_{j}=1-\prod_{i=1}^{N}\left(1-\psi_{i}\right)=1 \quad \text { on } \bigcup_{i=1}^{N} Q_{i}
$$

In order to estimate the partial derivatives of $\phi_{i}(i=1, \ldots, N)$ and their sum, we let

$$
\theta_{m}:=\sum_{i=1}^{m} \phi_{i}=1-\prod_{i=1}^{m}\left(1-\psi_{i}\right)
$$


Then $\phi_{1}=\theta_{1}$ and $\phi_{m}=\theta_{m}-\theta_{m-1}$ for $m=2, \ldots, N$. Since $\ell_{m} \leq \ell_{m-1}$, it suffices to show

$$
\left|\frac{\partial \theta_{m}}{\partial x_{k}}\right| \leq C \ell_{m}^{-1}, \quad\left|\frac{\partial^{2} \theta_{m}}{\partial x_{k}^{2}}\right| \leq C \ell_{m}^{-2}, \quad\left|\frac{\partial \theta_{m}}{\partial t}\right| \leq C \ell_{m}^{-2} .
$$

It is trivial that these are true outside $\bigcup_{i=1}^{m}(3 / 2) Q_{i}$. Fix $\boldsymbol{x} \in \bigcup_{i=1}^{m}(3 / 2) Q_{i}$. Then we easily check that

$$
\begin{aligned}
& \left|\frac{\partial \theta_{m}}{\partial x_{k}}(\boldsymbol{x})\right| \leq C \sum_{1 \leq i \leq m:(3 / 2) Q_{i} \ni \boldsymbol{x}} \ell_{i}^{-1}, \\
& \left|\frac{\partial \theta_{m}}{\partial t}(\boldsymbol{x})\right| \leq C \sum_{1 \leq i \leq m:(3 / 2) Q_{i} \ni \boldsymbol{x}} \ell_{i}^{-2}, \\
& \left|\frac{\partial^{2} \theta_{m}}{\partial x_{k}^{2}}(\boldsymbol{x})\right| \leq C\left\{\sum_{1 \leq i \leq m:(3 / 2) Q_{i} \ni \boldsymbol{x}} \ell_{i}^{-2}+\left(\sum_{1 \leq i \leq m:(3 / 2) Q_{i} \ni \boldsymbol{x}} \ell_{i}^{-1}\right)^{2}\right\},
\end{aligned}
$$

where a constant $C$ depends only on $n$. Since we can write $\ell_{i}=2^{\nu} \ell_{m}$ for some nonnegative integer $\nu$ if $i \leq m$ and the number of parabolic dyadic cubes $\widetilde{Q}$ of side length $\ell_{i}$ such that $(3 / 2) \widetilde{Q} \ni \boldsymbol{x}$ is at most $3^{n+1}$, it follows that

$$
\sum_{1 \leq i \leq m:(3 / 2) Q_{i} \ni \boldsymbol{x}} \ell_{i}^{-1} \leq 3^{n+1} \sum_{\nu=0}^{\infty}\left(2^{\nu} \ell_{m}\right)^{-1} \leq C \ell_{m}^{-1}
$$

Similarly, we have

$$
\sum_{1 \leq i \leq m:(3 / 2) Q_{i} \ni \boldsymbol{x}} \ell_{i}^{-2} \leq C \ell_{m}^{-2}
$$

Therefore we can obtain (3.5). Thus the lemma follows.

Lemma 3.6 Let $K$ be a compact set in $\mathbb{R}^{n+1}$ and let $r>0$. Then there exists a nonnegative function $\psi \in C^{\infty}\left(\mathbb{R}^{n+1}\right)$ such that $\psi=0$ on $K(r)$, $\psi=1$ on $\mathbb{R}^{n+1} \backslash K(3 \sqrt{n} r)$,

$$
\left|\frac{\partial \psi}{\partial x_{1}}\right|, \ldots,\left|\frac{\partial \psi}{\partial x_{n}}\right| \leq C r^{-1} \quad \text { on } \mathbb{R}^{n+1}
$$


and

$$
\left|\frac{\partial^{2} \psi}{\partial x_{1}^{2}}\right|, \ldots,\left|\frac{\partial^{2} \psi}{\partial x_{n}^{2}}\right|,\left|\frac{\partial \psi}{\partial t}\right| \leq C r^{-2} \quad \text { on } \mathbb{R}^{n+1}
$$

for some positive constant $C$ depending only on $n$.

Proof. Let $\left\{Q_{i}\right\}_{i=1}^{N}$ be a finite disjoint collection of parabolic dyadic cubes of side length $r$ such that $K(r) \subset \bigcup_{i=1}^{N} Q_{i}$ and $Q_{i} \cap K(r) \neq \emptyset$ for each $i$. By Lemma 3.5 , we find $\phi \in C_{0}^{\infty}\left(\mathbb{R}^{n+1}\right)$ such that $0 \leq \phi \leq 1$ on $\mathbb{R}^{n+1}, \phi=1$ on $K(r)$, spt $\phi \subset \bigcup_{i=1}^{N}(3 / 2) Q_{i} \subset K(3 \sqrt{n} r)$, and that (3.6) and (3.7) hold for $\phi$ instead of $\psi$. Then $\psi:=1-\phi$ is the required one.

For completeness of proofs of our theorems, we record the following two basic lemmas.

Lemma 3.7 Let $\ell=2^{-k}$ for some $k \in \mathbb{Z}$. Any parabolic cube of side length $\ell$ can be covered by at most $2^{n+1}$ mutually disjoint parabolic dyadic cubes of side length $\ell$.

Proof. Let $\left(x_{1}, \ldots, x_{n}, t\right) \in \mathbb{R}^{n+1}$. Then we find $\left(p_{1}, \ldots, p_{n+1}\right) \in \mathbb{Z}^{n+1}$ such that

$$
\left[x_{i}-\frac{1}{2} \ell, x_{i}+\frac{1}{2} \ell\right] \subset\left[\ell\left(p_{i}-1\right), \ell p_{i}\right] \cup\left[\ell p_{i}, \ell\left(p_{i}+1\right)\right] \quad(i=1, \ldots, n)
$$

and

$$
\left[t-\frac{1}{2} \ell^{2}, t+\frac{1}{2} \ell^{2}\right] \subset\left[\ell^{2}\left(p_{n+1}-1\right), \ell^{2} p_{n+1}\right] \cup\left[\ell^{2} p_{n+1}, \ell^{2}\left(p_{n+1}+1\right)\right] .
$$

By the definition of parabolic (dyadic) cubes, these yield the lemma.

Lemma 3.8 Let $k \geq j$ and let $Q_{1}$ and $Q_{2}$ be parabolic dyadic cubes of side length $2^{-k}$ and $2^{-j}$, respectively. If $Q_{1}$ and $Q_{2}$ are not disjoint, then $Q_{1} \subset Q_{2}$.

Proof. The case $k=j$ is trivial from definition. We consider the case $k>j$. Let

$$
Q_{1}=\prod_{i=1}^{n}\left[2^{-k} p_{i}, 2^{-k}\left(p_{i}+1\right)\right] \times\left[2^{-2 k} p_{n+1}, 2^{-2 k}\left(p_{n+1}+1\right)\right],
$$




$$
Q_{2}=\prod_{i=1}^{n}\left[2^{-j} q_{i}, 2^{-j}\left(q_{i}+1\right)\right] \times\left[2^{-2 j} q_{n+1}, 2^{-2 j}\left(q_{n+1}+1\right)\right],
$$

where $p_{i}, q_{i} \in \mathbb{Z}$ for $i=1, \ldots, n+1$. Note that $2^{-k}<2^{-j}$ in arguments below. Let $i=1, \ldots, n$. If $Q_{1}$ and $Q_{2}$ are not disjoint (i.e., $Q_{1} \cap Q_{2}$ has an interior), then at least one of the following holds:

$$
2^{-j} q_{i}<2^{-k} p_{i}<2^{-j}\left(q_{i}+1\right) \quad \text { or } 2^{-j} q_{i}<2^{-k}\left(p_{i}+1\right)<2^{-j}\left(q_{i}+1\right) .
$$

If the first case holds, then $p_{i}<2^{k-j}\left(q_{i}+1\right)$ and the both sides are integer. Therefore $p_{i}+1 \leq 2^{k-j}\left(q_{i}+1\right)$, and so $2^{-k}\left(p_{i}+1\right) \leq 2^{-j}\left(q_{i}+1\right)$. Hence

$$
\left[2^{-k} p_{i}, 2^{-k}\left(p_{i}+1\right)\right] \subset\left[2^{-j} q_{i}, 2^{-j}\left(q_{i}+1\right)\right] .
$$

If the second case holds, then $2^{k-j} q_{i}<p_{i}+1$ and the both sides are integer. Therefore $2^{-j} q_{i} \leq 2^{-k} p_{i}$, and so (3.8) holds. By the same reasoning as above, we have

$$
\left[2^{-2 k} p_{n+1}, 2^{-2 k}\left(p_{n+1}+1\right)\right] \subset\left[2^{-2 j} q_{n+1}, 2^{-2 j}\left(q_{n+1}+1\right)\right] .
$$

These imply that $Q_{1} \subset Q_{2}$.

\subsection{Polar sets and removability theorems}

A set $E$ in $\mathbb{R}^{n+1}$ is said to be polar if there exists a subcaloric function defined on a neighborhood of $E$ which takes $-\infty$ on $E$. Since $\mathcal{H}_{\mathrm{p}}^{n}(K) \leq$ $C \mathcal{M}_{\mathrm{p}}^{n}(K)$ for a compact set $K$, we can obtain the following lemma from [11, Theorem 1] and the fact that a countable union of polar sets is polar [13, Theorem 26].

Lemma 3.9 Let $E$ be a closed subset of $\mathbb{R}^{n+1}$ satisfying either $\mathcal{H}_{\mathrm{p}}^{n}(E)=0$ or $\mathcal{M}_{\mathrm{p}}^{n}(E)=0$. Then $E$ is polar.

For a function $v$ defined on $\Omega \backslash E$, we say that an inequality $v \leq C$ holds near $E$ if for each $\boldsymbol{y} \in E$ there is $r_{\boldsymbol{y}}>0$ such that $v(\boldsymbol{x}) \leq C$ for all $\boldsymbol{x} \in Q\left(\boldsymbol{y}, r_{\boldsymbol{y}}\right) \backslash E$. Lemma 3.9, together with [13, Theorem 29], yields the following.

Lemma 3.10 Let $E$ be a relatively closed subset of $\Omega$ satisfying either $\mathcal{H}_{\mathrm{p}}^{n}(E)=0$ or $\mathcal{M}_{\mathrm{p}}^{n}(E)=0$ and let $u$ be a subcaloric function on $\Omega \backslash E$. If $u$ is bounded above near $E$, then $u$ has a subcaloric extension to $\Omega$. 
We denote by $G_{\Omega}$ the Green function for the heat operator on $\Omega$. The following lemma may be known, but for the sake of the reader we provide a proof.

Lemma 3.11 Let $E$ be a relatively closed polar subset of $\Omega$. Then

$$
G_{\Omega \backslash E}=G_{\Omega} \quad \text { on }(\Omega \backslash E) \times(\Omega \backslash E) .
$$

In particular, if $E$ is a relatively closed subset of $\Omega$ satisfying either $\mathcal{H}_{\mathrm{p}}^{n}(E)=$ 0 or $\mathcal{M}_{\mathrm{p}}^{n}(E)=0$, then (3.9) holds.

Proof. Note that the Green function for $\Omega \backslash E$ is given by

$$
G_{\Omega \backslash E}(\boldsymbol{x}, \boldsymbol{y})=W(\boldsymbol{x}, \boldsymbol{y})-h_{\boldsymbol{y}}(\boldsymbol{x}),
$$

where $W$ is the fundamental solution of the heat equation and $h_{\boldsymbol{y}}$ is the greatest caloric minorant of $W(\cdot, \boldsymbol{y})$ on $\Omega \backslash E$ for each $\boldsymbol{y} \in \Omega \backslash E$. Thus $0 \leq h_{\boldsymbol{y}} \leq W(\cdot, \boldsymbol{y})$ on $\Omega \backslash E$, and so $h_{\boldsymbol{y}}$ is bounded near $E$. By [13, Theorem 29], $h_{\boldsymbol{y}}$ has a caloric extension $\bar{h}_{\boldsymbol{y}}$ to $\Omega$ and this is the greatest caloric minorant of $W(\cdot, \boldsymbol{y})$ on $\Omega$. Indeed, if $h$ is a caloric minorant of $W(\cdot, \boldsymbol{y})$ on $\Omega$, then $h \leq h_{\boldsymbol{y}}=\bar{h}_{\boldsymbol{y}}$ on $\Omega \backslash E$, and so $h \leq \bar{h}_{\boldsymbol{y}}$ on $\Omega$ by the continuity and $\mathfrak{m}(E)=0$. Thus the lemma follows.

\subsection{The distributional heat operator and the Riesz measure}

Following [14, Section 6.4], we recall the distributional heat operator and the Riesz measure. For simplicity, we use the symbols

$$
\Theta:=\Delta-\frac{\partial}{\partial t} \quad \text { and } \quad \Theta^{*}:=\Delta+\frac{\partial}{\partial t}
$$

to denote the heat operator and its adjoint operator, respectively. Let $u$ be a locally integrable function on $\Omega$. Define

$$
T_{u}(\phi):=\int_{\Omega} u \phi \mathrm{dm} \quad \text { for } \phi \in C_{0}^{\infty}(\Omega) .
$$

Then $T_{u}$ is a distribution in $\Omega$. The distributional heat operator of $u$ is defined by

$$
T_{\Theta u}(\phi):=\int_{\Omega} u \Theta^{*} \phi \mathrm{dm} \quad \text { for } \phi \in C_{0}^{\infty}(\Omega)
$$


If $T_{\Theta u}$ is a positive linear functional on $C_{0}^{\infty}(\Omega)$, then we see from the standard argument by approximation (cf. [14, the proof of Theorem 6.28]) that $T_{\Theta u}$ can be extended to $C_{0}(\Omega)$ as a positive linear functional. By the Riesz representation theorem, there exists a unique Radon measure $\mu_{u}$ on $\mathcal{B}(\Omega)$, the collection of Borel subsets of $\Omega$, such that

$$
T_{\Theta u}(\phi)=\int_{\Omega} \phi \mathrm{d} \mu_{u} \quad \text { for all } \phi \in C_{0}^{\infty}(\Omega) .
$$

We call $\mu_{u}$ the Riesz measure associated with $u$. Note from the uniqueness that if $D$ is an open subset of $\Omega$, then the restriction to $D$ of $\mu_{u}$ is the Riesz measure associated with $u$ on $D$.

Lemma 3.12 Let $u$ be a locally integrable function on $\Omega$. Then the following are equivalent:

(a) $T_{\Theta u}$ is a positive linear functional on $C_{0}^{\infty}(\Omega)$;

(b) there exists a subcaloric function $\bar{u}$ on $\Omega$ such that $\bar{u}=u \mathfrak{m}$-a.e. on $\Omega$.

Proof. The implication (b) to (a) follows from [14, Theorem 6.27]. We show the converse. Assume that (a) holds. Let $\mu_{u}$ be the Riesz measure associated with $u$ on $\Omega$ and let $D$ be an open set such that $\bar{D}$ is compact in $\Omega$. Define

$$
v(\boldsymbol{x}):=-\int_{D} W(\boldsymbol{x}, \boldsymbol{y}) \mathrm{d} \mu_{u}(\boldsymbol{y}) \quad \text { for } \boldsymbol{x} \in \mathbb{R}^{n+1},
$$

where $W$ is the Gauss-Weierstrass kernel on $\mathbb{R}^{n+1}$. Since $\mu_{u}(D)<\infty$, it follows from [13, Theorem 16] that $v$ is subcaloric on $\mathbb{R}^{n+1}$. In particular, $v$ is locally integrable on $\mathbb{R}^{n+1}$. By the Fubini theorem, $T_{\Theta(u-v)}(\phi)=T_{\Theta u}(\phi)-$ $T_{\Theta v}(\phi)=0$ for all $\phi \in C_{0}^{\infty}(D)$. By [5], there exists $h \in C^{\infty}(D)$ such that $\Theta h=0$ in $D$ in the classical sense and $T_{u-v}=T_{h}$ as a distribution in $D$. Therefore $u$ equals the subcaloric function $h+v \mathfrak{m}$-a.e. on $D$. Since $D$ is arbitrary, we obtain (b).

\section{Proof of Theorem 2.1}

This section presents the proof of Theorem 2.1.

Proof of Theorem 2.1. We first consider the case where $u$ is nonnegative. 
We see from upper semicontinuity and Lemma 3.4 that $u \in \mathcal{L}_{\text {loc }}^{1}(\Omega)$. Taking Lemmas 3.1 and 3.12 into account, it is enough to show that

$$
\int_{\Omega \backslash E} u \Theta^{*} \phi \mathrm{d} \mathfrak{m} \geq 0
$$

for all nonnegative functions $\phi \in C_{0}^{\infty}(\Omega)$, because any measurable extension to $\Omega$ of $u$ is locally integrable on $\Omega$ and its distributional heat operator is a positive linear functional on $C_{0}^{\infty}(\Omega)$. Let $\phi$ be such a test function and let $k \in \mathbb{N}$ be large enough. We take a finite disjoint collection of parabolic dyadic cubes $\left\{Q_{i}\right\}_{i=1}^{N}$ of side length $\ell=2^{-k}$ which covers spt $\phi$ and $(3 / 2) Q_{i} \subset \Omega$ for $i=1, \ldots, N$. Let $\left\{\phi_{i}\right\}_{i=1}^{N}$ be the partition of unity subordinate to those cubes with the properties in Lemma 3.5. We may suppose that

$$
\frac{3}{2} Q_{i} \cap E \neq \emptyset \quad \text { for } i=1, \ldots, M
$$

and

$$
\frac{3}{2} Q_{i} \cap E=\emptyset \quad \text { for } i=M+1, \ldots, N \text {. }
$$

Then $\phi \phi_{i}(i=M+1, \ldots, N)$ are nonnegative functions in $C_{0}^{\infty}(\Omega \backslash E)$. Since $u$ is subcaloric on $\Omega \backslash E$, we have

$$
\int_{\Omega \backslash E} u \Theta^{*}\left(\phi \phi_{i}\right) \mathrm{d} \mathfrak{m} \geq 0 \quad \text { for } i=M+1, \ldots, N \text {. }
$$

Noting $\phi=\sum_{i=1}^{N} \phi \phi_{i}$, we obtain

$$
\begin{aligned}
\int_{\Omega \backslash E} u \Theta^{*} \phi \mathrm{d} \mathfrak{m} & =\sum_{i=1}^{N} \int_{\Omega \backslash E} u \Theta^{*}\left(\phi \phi_{i}\right) \mathrm{d} \mathfrak{m} \\
& \geq \sum_{i=1}^{M} \int_{(3 / 2) Q_{i} \backslash E} u \Theta^{*}\left(\phi \phi_{i}\right) \mathrm{d} \mathfrak{m} .
\end{aligned}
$$

Since $\Delta\left(\phi \phi_{i}\right)=(\Delta \phi) \phi_{i}+2\left\langle\nabla \phi, \nabla \phi_{i}\right\rangle+\phi \Delta \phi_{i}$, we have

$$
\left|\Theta^{*}\left(\phi \phi_{i}\right)\right| \leq C \ell^{-2},
$$


where a constant $C$ depends only on $n$ and the maximum of $\phi$ and its derivatives. Let

$$
K:=\bigcup_{i=1}^{M} \frac{3}{2} Q_{i} \cap E .
$$

We observe that for each $i=1, \ldots, M$,

$$
\frac{3}{2} Q_{i} \subset K(3 \sqrt{n} \ell)
$$

and the cube $(3 / 2) Q_{i}$ intersects at most $3^{n+1}$ other cubes $(3 / 2) Q_{j}$, which implies

$$
\sum_{i=1}^{M} \chi_{(3 / 2) Q_{i}} \leq 3^{n+1} .
$$

Since $u$ is nonnegative, we have by (4.2) and (4.3)

$$
\begin{aligned}
\int_{\Omega \backslash E} u \Theta^{*} \phi \mathrm{d} \mathfrak{m} & \geq-C \ell^{-2} \sum_{i=1}^{M} \int_{(3 / 2) Q_{i} \backslash E} u \mathrm{~d} \mathfrak{m} \\
& \geq-C \ell^{-2} \int_{K(3 \sqrt{n} \ell) \backslash E} u \sum_{i=1}^{M} \chi_{(3 / 2) Q_{i}} \mathrm{~d} \mathfrak{m} \\
& \geq-C \ell^{-2} \int_{K(3 \sqrt{n} \ell) \backslash E} u \mathrm{dm} .
\end{aligned}
$$

Letting $\ell=2^{-k} \rightarrow 0$, we obtain (4.1) from Lemma 3.4. Thus $u$ has a subcaloric extension to $\Omega$.

At last, we remark on the case where $u$ is not necessarily nonnegative. Since $u^{+}$is subcaloric on $\Omega \backslash E$, the above argument shows that $u^{+}$has a subcaloric extension $\overline{u^{+}}$to $\Omega$. Then $u$ is bounded above near $E$ by virtue of the inequality $u \leq u^{+}$and the upper semicontinuity of $\overline{u^{+}}$on $\Omega$. It is easy to see that $\mathcal{M}_{\mathrm{p}}^{\alpha}(E)<\infty$ and $\alpha<n$ imply $\mathcal{M}_{\mathrm{p}}^{n}(E)=0$. Therefore we conclude from Lemma 3.10 that $u$ has a subcaloric extension to $\Omega$. This completes the proof. 


\section{Proof of Theorem 2.4}

In this section, we prove Theorem 2.4.

Proof of Theorem 2.4. As in the proof of Theorem 2.1, it is enough to prove (4.1) when $u$ is nonnegative. Let $\phi \in C_{0}^{\infty}(\Omega)$ be nonnegative, let $K:=\operatorname{spt} \phi$, and let $\varepsilon>0$ be sufficiently small so that $K(10 \sqrt{n} \varepsilon) \subset \Omega$. Write $A:=\overline{K(\varepsilon)} \cap E$. Since $\mathcal{H}_{\mathrm{p}, \varepsilon /(8 \sqrt{n})}^{\alpha}(A) \leq \mathcal{H}_{\mathrm{p}}^{\alpha}(E)<\infty$ and $A$ is compact, there are finitely many parabolic cubes $\left\{Q_{i}^{\prime}\right\}_{i=1}^{M}$ covering $A$ such that the side length of $Q_{i}^{\prime}$ is $\ell_{i}^{\prime} \leq \varepsilon /(8 \sqrt{n})$ for each $i$ and

$$
\sum_{i=1}^{M}\left(\ell_{i}^{\prime}\right)^{\alpha}<\mathcal{H}_{\mathrm{p}}^{\alpha}(E)+1
$$

Take $k_{i} \in \mathbb{Z}$ so that $2^{-k_{i}-1}<\ell_{i}^{\prime} \leq 2^{-k_{i}}$ and denote by $Q_{i}^{\prime \prime}$ the parabolic cube with the same center as $Q_{i}^{\prime}$ and side length $2^{-k_{i}}$. Then $Q_{i}^{\prime} \subset Q_{i}^{\prime \prime}$. By Lemma 3.7 , each $Q_{i}^{\prime \prime}$ is covered by at most $2^{n+1}$ mutually disjoint parabolic dyadic cubes of side length $2^{-k_{i}}$. Note from Lemma 3.8 that two parabolic dyadic cubes are either disjoint or one is contained in the other. Removing extra cubes contained in the other if necessary, we can find a finite disjoint collection of parabolic dyadic cubes $\left\{Q_{i}\right\}_{i=1}^{N}$, where $N \leq 2^{n+1} M$, such that this covers $A, Q_{i} \cap A \neq \emptyset(i=1, \ldots, N)$ and the side length of $Q_{i}$ is $\ell_{i} \leq 2 \max \left\{\ell_{1}^{\prime}, \ldots, \ell_{M}^{\prime}\right\} \leq \varepsilon /(4 \sqrt{n})(i=1, \ldots, N)$. Then

$$
\sum_{i=1}^{N} \ell_{i}^{\alpha} \leq 2^{n+1} \sum_{i=1}^{M}\left(2 \ell_{i}^{\prime}\right)^{\alpha}<2^{n+1+\alpha}\left(\mathcal{H}_{\mathrm{p}}^{\alpha}(E)+1\right)<\infty
$$

For each $i=1, \ldots, N$, there are $3^{n+1}-1$ many parabolic dyadic cubes $\left\{Q_{i}^{j_{i}}\right\}$ which are adjacent to $Q_{i}$ and have the same side length $\ell_{i}$ as $Q_{i}$. As above, we can take away extra cubes contained in other one so that $\left\{Q_{i}, Q_{i}^{j_{i}}\right\}_{i, j_{i}}$ is a maximal disjoint collection. Let $\ell:=\min \left\{\ell_{1}, \ldots, \ell_{N}\right\}$ and let $\left\{\widetilde{Q}_{k}^{\prime}\right\}$ be a finitely many disjoint collection of parabolic dyadic cubes of side length $\ell$ such that $K \subset \bigcup_{k} \widetilde{Q}_{k}^{\prime}$ and $\widetilde{Q}_{k}^{\prime} \cap K \neq \emptyset$ for each $k$. By Lemma 3.8, one of the following holds:

$$
\widetilde{Q}_{k}^{\prime} \subset \bigcup_{i} Q_{i} \cup\left(\bigcup_{i} \bigcup_{j_{i}} Q_{i}^{j_{i}}\right) \quad \text { or } \quad \widetilde{Q}_{k}^{\prime} \cap\left(\bigcup_{i} Q_{i} \cup\left(\bigcup_{i} \bigcup_{j_{i}} Q_{i}^{j_{i}}\right)\right)=\emptyset \text {. }
$$


Let $\left\{\widetilde{Q}_{k}\right\}$ be the collection of all $\widetilde{Q}_{k}^{\prime}$ satisfying the second one. Then $\left\{\widetilde{Q}_{k}\right\}$ is a finite disjoint collection of parabolic dyadic cubes of side length $\ell$ such that $\left\{Q_{i}, Q_{i}^{j_{i}}, \widetilde{Q}_{k}\right\}$ are mutually disjoint, $\widetilde{Q}_{k} \cap K \neq \emptyset$ for each $k$ and

$$
K \backslash\left[\bigcup_{i} Q_{i} \cup\left(\bigcup_{i} \bigcup_{j_{i}} Q_{i}^{j_{i}}\right)\right] \subset \bigcup_{k} \widetilde{Q}_{k} .
$$

We see that

$$
\frac{3}{2} \widetilde{Q}_{k} \subset \mathbb{R}^{n+1} \backslash\left(\bigcup_{i=1}^{N} Q_{i}\right) \subset \mathbb{R}^{n+1} \backslash A .
$$

Indeed, denoting by $3 Q_{i}$ the parabolic cube with the same center as $Q_{i}$ and side lengths $3 \ell_{i}$ in the spatial direction and $3 \ell_{i}^{2}$ in the temporal direction as defined in (3.4), we have $3 Q_{i} \subset \bigcup_{i} Q_{i} \cup\left(\bigcup_{i} \bigcup_{j_{i}} Q_{i}^{j_{i}}\right)$ since $3 Q_{i}$ coincides with the union of $Q_{i}$ and all parabolic dyadic cubes of side length $\ell_{i}$ which are adjacent to $Q_{i}$. Therefore $3 Q_{i}$ and $\widetilde{Q}_{k}$ are disjoint. Since $\ell \leq \ell_{i}$, it follows that $Q_{i}$ and $3 \widetilde{Q}_{k}$ are disjoint, and so $(3 / 2) \widetilde{Q}_{k} \subset \mathbb{R}^{n+1} \backslash Q_{i}$. Moreover, if $\boldsymbol{x} \in(3 / 2) \widetilde{Q}_{k}, \boldsymbol{y} \in E \backslash \overline{K(\varepsilon)}$ and $\boldsymbol{z} \in \widetilde{Q}_{k} \cap K$, then

$$
d_{\mathrm{p}}(\boldsymbol{x}, \boldsymbol{y}) \geq d_{\mathrm{p}}(\boldsymbol{y}, \boldsymbol{z})-d_{\mathrm{p}}(\boldsymbol{x}, \boldsymbol{z}) \geq \varepsilon-3 \sqrt{n} \ell>0 .
$$

Hence $(3 / 2) \widetilde{Q}_{k} \cap E=\emptyset$ for all $k$. Let $\left\{\phi_{i}, \phi_{i}^{j_{i}}, \widetilde{\phi}_{k}\right\}$ be the partition of unity subordinate to $\left\{Q_{i}, Q_{i}^{j_{i}}, \widetilde{Q}_{k}\right\}$ with the properties in Lemma 3.5. Since $u$ is subcaloric on $\Omega \backslash E$, we have

$$
\int_{\Omega \backslash E} u \Theta^{*}\left(\phi \widetilde{\phi}_{k}\right) \mathrm{d} \mathfrak{m} \geq 0 \quad \text { for each } k .
$$

For simplicity, we write $\psi:=\sum_{i} \phi_{i}+\sum_{i} \sum_{j_{i}} \phi_{i}^{j_{i}}$. Then

$$
\begin{aligned}
\int_{\Omega \backslash E} u \Theta^{*} \phi \mathrm{d} \mathfrak{m} & =\int_{\Omega \backslash E} u \Theta^{*}\left[\phi\left(\psi+\sum_{k} \widetilde{\phi}_{k}\right)\right] \mathrm{d} \mathfrak{m} \\
& \geq \int_{D \backslash E} u \Theta^{*}(\phi \psi) \mathrm{d} \mathfrak{m},
\end{aligned}
$$

where $D:=\bigcup_{i}(3 / 2) Q_{i} \cup\left(\bigcup_{i} \bigcup_{j_{i}}(3 / 2) Q_{i}^{j_{i}}\right)$, the union for parabolic dyadic 
cubes in $\left\{Q_{i}, Q_{i}^{j_{i}}\right\}_{i, j_{i}}$. Note that $D \subset A(8 \sqrt{n} \varepsilon)$ since $Q_{i} \cap A \neq \emptyset$. In order to estimate the last integral in (5.2), let us split $D$ into mutually disjoint sets. By relabeling, we may assume that $\ell_{1} \geq \ell_{2} \geq \cdots \geq \ell_{N}$. For each $i=1, \ldots, N$, let $R_{i}:=(3 / 2) Q_{i} \cup\left(\bigcup_{j_{i}}(3 / 2) Q_{i}^{j_{i}}\right)$, where the union is for parabolic dyadic cubes in $\left\{Q_{i}^{j_{i}}\right\}_{j_{i}}$, and let

$$
\widetilde{R}_{i}:=R_{i} \backslash\left(\bigcup_{k=i+1}^{N} R_{k}\right)
$$

Then $\left\{\widetilde{R}_{i}\right\}_{i=1}^{N}$ are mutually disjoint, $\widetilde{R}_{i} \subset R_{i}$ for each $i$ and $\bigcup_{i=1}^{N} \widetilde{R}_{i}=D$. Moreover, if $\boldsymbol{x} \in \widetilde{R}_{m}$, then $\phi_{i}(\boldsymbol{x}), \phi_{i}^{j_{i}}(\boldsymbol{x})=0$ for all $i>m$ and $j_{i}$, and so

$$
\psi(\boldsymbol{x})=\sum_{i \leq m} \phi_{i}(\boldsymbol{x})+\sum_{i \leq m} \sum_{j_{i}} \phi_{i}^{j_{i}}(\boldsymbol{x}) .
$$

Therefore $\left|\Theta^{*}(\phi \psi)\right| \leq C \ell_{m}^{-2}$ on $\widetilde{R}_{m}$. Let $q_{\alpha}^{\prime}$ be the Hölder conjugate exponent of $q_{\alpha}$. Since $-2 q_{\alpha}^{\prime}+n+2=\alpha$, we have by (5.1)

$$
\int_{D}\left|\Theta^{*}(\phi \psi)\right|^{q_{\alpha}^{\prime}} \mathrm{d} \mathfrak{m}=\sum_{i} \int_{\widetilde{R}_{i}}\left|\Theta^{*}(\phi \psi)\right|^{q_{\alpha}^{\prime}} \mathrm{d} \mathfrak{m} \leq C \sum_{i} \ell_{i}^{-2 q_{\alpha}^{\prime}+n+2} \leq C .
$$

Hence, by the Hölder inequality,

$$
\begin{aligned}
\int_{\Omega \backslash E} u \Theta^{*} \phi \mathrm{d} \mathfrak{m} & \geq \int_{D \backslash E} u \Theta^{*}(\phi \psi) \mathrm{d} \mathfrak{m} \\
& \geq-\left(\int_{D \backslash E} u^{q_{\alpha}} \mathrm{d} \mathfrak{m}\right)^{1 / q_{\alpha}}\left(\int_{D}\left|\Theta^{*}(\phi \psi)\right|^{q_{\alpha}^{\prime}} \mathrm{d} \mathfrak{m}\right)^{1 / q_{\alpha}^{\prime}} \\
& \geq-C\left(\int_{A(8 \sqrt{n} \varepsilon) \backslash E} u^{q_{\alpha}} \mathrm{d} \mathfrak{m}\right)^{1 / q_{\alpha}}
\end{aligned}
$$

Since $u \in \mathcal{L}_{\text {loc }}^{q_{\alpha}}(\Omega)$ and $\mathfrak{m}(A(8 \sqrt{n} \varepsilon) \backslash E) \rightarrow 0$, the last integral tends to 0 as $\varepsilon \rightarrow 0$. Hence we obtain (4.1). The rest of the proof is the same as that of Theorem 2.1. 


\section{Proofs of Corollaries 2.6-2.8}

Proof of Corollary 2.6. Since $u$ is a nonnegative subcaloric function on $\Omega \backslash E$, it has a subcaloric extension to $\Omega$ by Theorem 2.1. In particular, $u \in \mathcal{L}_{\text {loc }}^{\infty}(\Omega)$. Let $D$ be a bounded open set whose closure is compact in $\Omega$. By the Riesz decomposition theorem [13, Theorem 22] with $\mathrm{d} \mu=\Theta u \mathrm{dm}=$ $u^{q} \mathrm{~d} \mathfrak{m}$ (since $u$ is $C^{2,1}$ ) and Lemma 3.11, we have for all $\boldsymbol{x} \in D \backslash E$,

$$
u(\boldsymbol{x})=h(\boldsymbol{x})-\int_{D \backslash E} G_{D}(\boldsymbol{x}, \boldsymbol{y}) u(\boldsymbol{y})^{q} \mathrm{~d} \mathfrak{m}(\boldsymbol{y}),
$$

where $G_{D}$ is the Green function for $\Theta$ on $D$ and $h$ is the smallest caloric majorant of $u$ on $D \backslash E$. Since $u$ is bounded on $D \backslash E$, so is $h$. Therefore $h$ has a caloric extension $\bar{h}$ to $D$. For $\boldsymbol{x} \in D$, we define

$$
\bar{u}(\boldsymbol{x}):=\bar{h}(\boldsymbol{x})-\int_{D \backslash E} G_{D}(\boldsymbol{x}, \boldsymbol{y}) u(\boldsymbol{y})^{q} \mathrm{~d} \mathfrak{m}(\boldsymbol{y}) .
$$

Then $\bar{u}=u$ on $D \backslash E$. By the regularity theorem for Green potentials (cf. [2, p. 303]) and $q \geq 1$, we see that $\bar{u}$ is a $C^{2,1}$-function on $D$. By the FubiniTonelli theorem, we also have $\Theta \bar{u}=\bar{u}^{q}$ in $D$ in the sense of distributions. These imply that $\bar{u}$ is a classical solution of $\Theta \bar{u}=\bar{u}^{q}$ in $D$. Since $D$ is arbitrary, we obtain Corollary 2.6.

Proof of Corollary 2.7. By Theorem 2.4, $u$ has a subcaloric extension to $\Omega$. The rest of the proof is the same as the proof of Corollary 2.6.

For the proof of Corollary 2.8, we need the following a priori estimate.

Lemma 6.1 The assumptions are the same as in Corollary 2.8. Then there exists a positive constant $C$ depending only on $q$ and $n$ such that

$$
u(\boldsymbol{x}) \leq C d_{\mathrm{p}}(\boldsymbol{x}, E)^{-2 /(q-1)} \quad \text { near } E .
$$

Proof. The proof is based on Véron's comparison argument [12, Lemma 1]. Let $\boldsymbol{y} \in E$ and take $r_{\boldsymbol{y}}>0$ so that $d_{\mathrm{p}}(\boldsymbol{x}, E) \leq d_{\mathrm{p}}(\boldsymbol{x}, \partial \Omega)$ for all $\boldsymbol{x} \in Q\left(\boldsymbol{y}, r_{\boldsymbol{y}}\right)$. Fix $\boldsymbol{x}_{0}=\left(x_{0}, t_{0}\right) \in Q\left(\boldsymbol{y}, r_{\boldsymbol{y}}\right) \backslash E$ and let $R:=d_{\mathrm{p}}\left(\boldsymbol{x}_{0}, E\right) / 4$ and $t_{1}:=t_{0}-R^{2}$. We consider a function on $D:=\left\{(x, t):\left\|x-x_{0}\right\|<R, t_{1}<t<t_{0}\right\} \subset \Omega \backslash E$ defined by 


$$
v(x, t):=\lambda\left\{\left(R^{2}-\left\|x-x_{0}\right\|^{2}\right)^{-2 /(q-1)}+\left(t-t_{1}\right)^{-2 /(q-1)}\right\},
$$

where $\lambda$ is a positive constant chosen later. Letting $r:=\left\|x-x_{0}\right\|$, it is easy to check that

$$
\begin{aligned}
& \Delta v=\frac{4 \lambda}{(q-1)^{2}}\left(R^{2}-r^{2}\right)^{-2 q /(q-1)}\left\{n(q-1) R^{2}+(n+2-q(n-2)) r^{2}\right\}, \\
& \frac{\partial v}{\partial t}=-\frac{2 \lambda}{q-1}\left(t-t_{1}\right)^{-(q+1) /(q-1)} .
\end{aligned}
$$

Since $v^{q} \geq \lambda^{q}\left\{\left(R^{2}-r^{2}\right)^{-2 q /(q-1)}+\left(t-t_{1}\right)^{-2 q /(q-1)}\right\}$, we have

$$
\begin{aligned}
\frac{\partial v}{\partial t}-\Delta v+v^{q} \geq & \lambda\left(R^{2}-r^{2}\right)^{-2 q /(q-1)} \\
& \times\left[\lambda^{q-1}-\frac{4}{(q-1)^{2}}\left\{n(q-1) R^{2}+(n+2-q(n-2)) r^{2}\right\}\right] \\
& +\lambda\left(t-t_{1}\right)^{-2 q /(q-1)}\left\{\lambda^{q-1}-\frac{2}{q-1}\left(t-t_{1}\right)\right\} .
\end{aligned}
$$

If we choose

$$
\lambda:=\left(\max \left\{\frac{8(q+1)}{(q-1)^{2}}, \frac{4 n}{q-1}\right\} R^{2}\right)^{1 /(q-1)}
$$

then $\partial v / \partial t-\Delta v+v^{q} \geq 0$ in $D$, and therefore we have by $[1$, Lemma 1$]$

$$
\begin{aligned}
\Delta(u-v)^{+}-\frac{\partial(u-v)^{+}}{\partial t} & \geq \operatorname{sign}^{+}(u-v) \cdot\left(\Delta(u-v)-\frac{\partial(u-v)}{\partial t}\right) \\
& \geq \operatorname{sign}^{+}(u-v) \cdot\left(u^{q}-v^{q}\right) \\
& \geq 0
\end{aligned}
$$

in $D$ in the sense of distributions, where

$$
\operatorname{sign}^{+} a= \begin{cases}1 & (a>0), \\ 1 / 2 & (a=0), \\ 0 & (a<0)\end{cases}
$$


Since $(u-v)^{+}=0$ near the parabolic boundary of $D$, it follows from the maximum principle that $(u-v)^{+}=0$ on $D$, and so

$$
u\left(\boldsymbol{x}_{0}\right) \leq v\left(\boldsymbol{x}_{0}\right)=2 \lambda R^{-4 /(q-1)} \leq C(q, n) R^{-2 /(q-1)} .
$$

Since $R=d_{\mathrm{p}}\left(\boldsymbol{x}_{0}, E\right) / 4$, the lemma is proved.

Lemma 6.2 The assumptions are the same as in Corollary 2.8. Then $u \in \mathcal{L}_{\text {loc }}^{q}(\Omega)$.

Proof. Let $\boldsymbol{y} \in E$. By Lemma 6.1 and $q \geq q_{\alpha}$, there is $\ell:=r_{\boldsymbol{y}}>0$ small enough such that $4 Q \subset \Omega$ and

$$
u(\boldsymbol{x}) \leq C d_{\mathrm{p}}(\boldsymbol{x}, E)^{-2 /(q-1)} \leq C d_{\mathrm{p}}(\boldsymbol{x}, E)^{\alpha-n} \quad \text { for all } \boldsymbol{x} \in 2 Q \backslash E,
$$

where $Q:=Q(\boldsymbol{y}, \ell)$ and $4 Q$ is defined in the same way as in (3.4). Take a nonnegative function $\phi \in C_{0}^{\infty}\left(\mathbb{R}^{n+1}\right)$ so that $\phi=1$ on $Q$, spt $\phi \subset 2 Q$,

$$
\left|\frac{\partial \phi}{\partial x_{1}}\right|, \ldots,\left|\frac{\partial \phi}{\partial x_{n}}\right| \leq C \ell^{-1} \quad \text { on } \mathbb{R}^{n+1}
$$

and

$$
\left|\frac{\partial^{2} \phi}{\partial x_{1}^{2}}\right|, \ldots,\left|\frac{\partial^{2} \phi}{\partial x_{n}^{2}}\right|,\left|\frac{\partial \phi}{\partial t}\right| \leq C \ell^{-2} \quad \text { on } \mathbb{R}^{n+1}
$$

Let $0<r<\ell$ and $K:=2 Q \cap E$. Take $\psi_{r} \in C^{\infty}\left(\mathbb{R}^{n+1}\right)$ with the properties in Lemma 3.6. Then $\phi \psi_{r} \in C_{0}^{\infty}(2 Q \backslash E)$. Since $\Theta u=u^{q}$ in $\Omega \backslash E$ and $\left|\Theta^{*} \psi_{r}\right|=\left|\nabla \psi_{r}\right|=0$ on $2 Q \backslash K(3 \sqrt{n} r)$, we have

$$
\begin{aligned}
\int_{Q \backslash K(3 \sqrt{n} r)} u^{q} \mathrm{~d} \mathfrak{m} & \leq \int_{2 Q \backslash E} \phi \psi_{r} u^{q} \mathrm{~d} \mathfrak{m}=\int_{2 Q \backslash E} u \Theta^{*}\left(\phi \psi_{r}\right) \mathrm{d} \mathfrak{m} \\
& \leq C \ell^{-2} \int_{2 Q \backslash E} u \mathrm{~d} \mathfrak{m}+C r^{-2} \int_{(2 Q \cap K(3 \sqrt{n} r)) \backslash E} u \mathrm{~d} \mathfrak{m} .
\end{aligned}
$$

Combining (6.1), (6.2) and Lemma 3.3, we find a constant $C$ independent of $r$ such that

$$
\int_{Q \backslash K(3 \sqrt{n} r)} u^{q} \mathrm{~d} \mathfrak{m} \leq C .
$$


Letting $r \rightarrow 0$, we have $\int_{Q \backslash E} u^{q} \mathrm{dm} \leq C$. The standard argument by a finite covering and the continuity of $u$ on $\Omega \backslash E$ conclude that $u \in \mathcal{L}_{\text {loc }}^{q}(\Omega)$.

Proof of Corollary 2.8. From Lemma 6.2 and Theorem 2.4, we see that $u$ has a subcaloric extension to $\Omega$. The rest of the proof is the same as the proof of Corollary 2.6.

\section{Appendix}

\subsection{Removable set considered by Takahashi and Yanagida}

Let $m \in \mathbb{N}$ be such that $1 \leq m<n-2$ and let $T>0$. In [10], Takahashi and Yanagida discussed the removability of the following set:

$$
E:=\left\{(\xi(s, t), t):(s, t) \in[0,1]^{m} \times[0, T]\right\} \subset \mathbb{R}^{n+1},
$$

where

$$
\xi(s, t)=\left(\xi^{1}(s, t), \ldots, \xi^{n}(s, t)\right):[0,1]^{m} \times[0, T] \rightarrow \mathbb{R}^{n}
$$

is continuously differentiable with respect to $s=\left(s_{1}, \ldots, s_{m}\right)$ and $1 / 2$ Hölder continuous with respect to $t$ uniformly for $s$ such that

$$
\operatorname{rank}\left(\begin{array}{ccc}
\xi_{s_{1}}^{1}(s, t) & \cdots & \xi_{s_{m}}^{1}(s, t) \\
\vdots & \ddots & \vdots \\
\xi_{s_{1}}^{n}(s, t) & \cdots & \xi_{s_{m}}^{n}(s, t)
\end{array}\right)=m
$$

for any $(s, t) \in[0,1]^{m} \times[0, T]$. More generally, we have the following.

Proposition 7.1 Notations are the same as above. If $\xi(s, t)$ is Lipschitz continuous with respect to $s$ uniformly for $t$ and $1 / 2$-Hölder continuous with respect to $t$ uniformly for $s$, then $\mathcal{M}_{\mathrm{p}}^{m+2}(E)<\infty$.

Proof. For simplicity, we write $F(s, t):=(\xi(s, t), t)$ and $R:=[0,1]^{m} \times$ $[0, T]$. Then $E=F(R)$ and there exists a constant $C_{2}>1$ such that for all $\left(s_{1}, t_{1}\right),\left(s_{2}, t_{2}\right) \in R$

$$
\begin{aligned}
d_{\mathrm{p}}\left(F\left(\boldsymbol{s}_{1}, t_{1}\right), F\left(\boldsymbol{s}_{2}, t_{2}\right)\right) & =\left\|\xi\left(\boldsymbol{s}_{1}, t_{1}\right)-\xi\left(\boldsymbol{s}_{2}, t_{2}\right)\right\|+\left|t_{1}-t_{2}\right|^{1 / 2} \\
& \leq C_{2}\left(\left\|\boldsymbol{s}_{1}-\boldsymbol{s}_{2}\right\|_{\mathbb{R}^{m}}+\left|t_{1}-t_{2}\right|^{1 / 2}\right)
\end{aligned}
$$


Let $r>0$ be small and let $\left\{\widetilde{Q}_{i}\right\}_{i=1}^{N}$ be a finite disjoint collection of parabolic dyadic cubes in $\mathbb{R}^{m} \times \mathbb{R}$ of side length $r$ such that $R \subset \bigcup_{i=1}^{N} \widetilde{Q}_{i}$ and $\widetilde{Q}_{i} \cap R \neq \emptyset$ for each $i$. Then $N \leq \mathrm{Cr}^{-m-2}$. Also, we see from (7.1) that for each $i$, there exists a parabolic cube $Q_{i}$ in $\mathbb{R}^{n+1}$ of side length $2 \sqrt{m} C_{2} r$ such that $F\left(\widetilde{Q}_{i} \cap R\right) \subset Q_{i}$, and so $E \subset \bigcup_{i=1}^{N} Q_{i}$. For each $i$, let $\left\{Q_{i}^{j_{i}}\right\}_{j_{i}}$ be $3^{n+1}-1$ many parabolic cubes in $\mathbb{R}^{n+1}$ of side length $2 \sqrt{m} C_{2} r$ which are adjacent to $Q_{i}$. Then $E(r) \subset \bigcup_{i=1}^{N}\left(Q_{i} \cup\left(\bigcup_{j_{i}} Q_{i}^{j_{i}}\right)\right)$. Therefore

$$
\mathfrak{m}(E(r)) \leq 3^{n+1} \sum_{i=1}^{N} \mathfrak{m}\left(Q_{i}\right) \leq C N r^{n+2} \leq C r^{n-m} .
$$

Hence $\mathcal{M}_{\mathrm{p}}^{m+2}(E)<\infty$.

\subsection{Results for semilinear elliptic equations}

We record some removability theorems for subharmonic functions and positive solutions of a semilinear elliptic equation with an absorption term:

$$
\Delta u=u^{q},
$$

where $q \geq 1$. In below, $\mathcal{M}^{\alpha}$ and $\mathcal{H}^{\alpha}$ stand for the usual $\alpha$-dimensional upper Minkowski content and Hausdorff measure on $\mathbb{R}^{n}$ (with respect to the Euclidean distance). Let $q_{\alpha}^{*}:=(n-\alpha) /(n-\alpha-2)$. In the same way as above, we can prove the following results.

Theorem 7.2 Let $0 \leq \alpha<n-2$, let $\Omega$ be an open set in $\mathbb{R}^{n}$, let $E$ be a relatively closed subset of $\Omega$ such that $\mathcal{H}^{\alpha}(E)<\infty$, and let $u$ be a subharmonic function on $\Omega \backslash E$. If $u^{+} \in \mathcal{L}_{\text {loc }}^{q_{\alpha}^{*}}(\Omega)$, then $u$ has a subharmonic extension to $\Omega$.

A result for subharmonic functions corresponding to Theorem 2.1 can be found in [9]. Thus we can obtain the following results for (7.2).

Corollary 7.3 Let $0 \leq \alpha<n-2$, let $\Omega$ be an open set in $\mathbb{R}^{n}$, let $E$ be a relatively closed subset of $\Omega$, and let $u$ be a nonnegative $C^{2}$-solution of (7.2) in $\Omega \backslash E$. Assume one of the following:

(i) $\mathcal{M}^{\alpha}(E)=0$ and

$$
\limsup _{\Omega \backslash E \ni x \rightarrow y} d(x, E)^{n-\alpha-2} u(x)<\infty \quad \text { for each } y \in E ;
$$


(ii) $\mathcal{M}^{\alpha}(E)<\infty$ and

$$
\lim _{\Omega \backslash E \ni x \rightarrow y} d(x, E)^{n-\alpha-2} u(x)=0 \quad \text { for each } y \in E .
$$

Then $u$ can be extended to the whole of $\Omega$ as a $C^{2}$-solution of (7.2) in $\Omega$.

Corollary 7.4 Let $0 \leq \alpha<n-2$, let $\Omega$ be an open set in $\mathbb{R}^{n}$, let $E$ be a relatively closed subset of $\Omega$ such that $\mathcal{H}^{\alpha}(E)<\infty$, and let $u$ be a nonnegative $C^{2}$-solution of (7.2) in $\Omega \backslash E$. If $u \in \mathcal{L}_{\text {loc }}^{q_{\alpha}^{*}}(\Omega)$, then $u$ can be extended to the whole of $\Omega$ as a $C^{2}$-solution of (7.2) in $\Omega$.

Corollary 7.5 Let $0 \leq \alpha<n-2$, let $\Omega$ be an open set in $\mathbb{R}^{n}$, let $E$ be a relatively closed subset of $\Omega$, let

$$
q \geq \frac{n-\alpha}{n-\alpha-2}
$$

and let $u$ be a nonnegative $C^{2}$-solution of $(7.2)$ in $\Omega \backslash E$. If $\mathcal{M}^{\alpha}(E)<\infty$, then $u$ can be extended to the whole of $\Omega$ as a $C^{2}$-solution of (7.2) in $\Omega$.

Acknowledgement The author would like to thank the referee and the editor for reading carefully and giving several comments.

\section{References}

[ 1 ] Brézis H. and Véron L., Removable singularities for some nonlinear elliptic equations. Arch. Rational Mech. Anal. 75 (1980/81), 1-6.

[2] Doob J. L., Classical potential theory and its probabilistic counterpart. Springer-Verlag, 2001.

[ 3 ] Gardiner S. J., Removable singularities for subharmonic functions. Pacific J. Math. 147 (1991), 71-80.

[ 4 ] Harvey R. and Polking J., Removable singularities of solutions of linear partial differential equations. Acta Math. 125 (1970), 39-56.

[ 5 ] Hörmander L., Linear partial differential operators. Springer-Verlag New York Inc., 1969.

[ 6 ] Hirata K., Removable singularities of semilinear parabolic equations. Proc. Amer. Math. Soc. 142 (2014), 157-171.

[ 7 ] Hsu S. Y., Removable singularities of semilinear parabolic equations. Adv. Differential Equations 15 (2010), 137-158.

[8] Hui K. M., Another proof for the removable singularities of the heat equa- 
tion. Proc. Amer. Math. Soc. 138 (2010), 2397-2402.

[ 9 ] Riihentaus J., Removable sets for subharmonic functions. Pacific J. Math. 194 (2000), 199-208.

[10] Takahashi J. and Yanagida E., Removability of time-dependent singularities in the heat equation. preprint (http://arxiv.org/abs/1304.5147).

[11] Taylor S. J. and Watson N. A., A Hausdorff measure classification of polar sets for the heat equation. Math. Proc. Cambridge Philos. Soc. 97 (1985), 325-344.

[12] Véron L., Singularités éliminables d'équations elliptiques non linéaires. J. Differential Equations 41 (1981), 87-95.

[13] Watson N. A., Green functions, potentials, and the Dirichlet problem for the heat equation. Proc. London Math. Soc. (3) 33 (1976), 251-298.

[14] Watson N. A., Introduction to heat potential theory. Mathematical Surveys and Monographs 182, American Mathematical Society, Providence, RI, 2012 .

\author{
Department of Mathematics \\ Graduate School of Science \\ Hiroshima University \\ Higashi-Hiroshima 739-8526, Japan \\ E-mail: hiratake@hiroshima-u.ac.jp
}

